\title{
Predicción de parada de máquinas generadoras en una central hidroeléctrica por medio de minería de datos
}

\author{
Herson A. González¹, Juan D. Piedrahita ${ }^{1}$ y Omar D. Castrillón 1 \\ (1) Universidad Nacional de Colombia, Facultad de Ingeniería y Arquitectura, Departamento de Ingeniería \\ Industrial, Campus La Nubia Bloque Q piso 2, Manizales - Colombia. \\ (correo-e: heagonzalezpr@unal.edu.co,jdpiedrahitas@unal.edu.co,odcastrillong@unal.edu.co)
}

Recibido Abr. 20, 2020; Aceptado Jun. 17, 2020; Versión final Jul. 15, 2020, Publicado Oct. 2020

\section{Resumen}

Se desarrolla una metodología para predecir la parada de las máquinas generadoras en una central hidroeléctrica por medio de la plataforma Weka y el algoritmo de clasificación J48. Se construye un archivo con 300 registros reales y 11 variables que incluyen una variable dependiente (paro de máquina) y 10 variables independientes: temperatura cojinete guía superior, flujo de agua intercambiadores de calor, presión de regulación de velocidad, presión tubería de carga, caudal tubería de carga, nivel de embalse, carga generada, frecuencia, temperatura cojinete guía turbina, y clima. Usando la herramienta XrealStats, se realiza un análisis de correlación (Pearson) entre cada una de las variables independientes y la variable dependiente. Como resultado se logra predecir los disparos de las máquinas con un acierto del $94 \%$. Se concluye que el árbol de clasificación obtenido en esta investigación permite predecir fácilmente futuros paros de máquinas con un acierto superior al $94 \%$.

Palabras clave: minería de datos; Bayesiano; planillas de control, central hidroeléctrica, maquinas generadoras

\section{Prediction of generating machine shutdowns at a hydroelectric power station by data mining}

\begin{abstract}
A methodology is developed to predict shutdowns of generating machines in a hydroelectric power station by using the platform Weka and the algorithm J48. A file was built with 300 real data registrations and 11 variables consisting of a single dependent variable (machine trip) and ten independent variables: temperature, water flow, regulatory pressure, pipeline pressure, flow rate of the reservoir level, generated load, frequency, oil temperature, and climate. Using XRealStats tools, a Pearson correlation analysis was performed between each of the independent variables and the dependent variable. The results showed that it is possible to predict machine trips with a success rate of $94 \%$. It is concluded that the classification tree generated in this research predicts future machine trips with over $94 \%$ accuracy.
\end{abstract}

Keywords: data mining; Bayesian; control sheets; hydroelectric power station; generating machines 


\section{INTRODUCCIÓN}

En Colombia las empresas generadoras no tienen una responsabilidad directa sobre la planificación de la capacidad productiva a largo plazo, esta responsabilidad recae sobre la unidad de planeación minero energética del gobierno nacional (https://www1.upme.gov.co/Paginas/default.aspx) adscrita al Ministerio de Minas y Energía de Colombia. Las hidroeléctricas actúan, como un agente de mercado a través de sus casas matrices, adoptando decisiones de operación, mantenimiento o inversión que se enmarquen en sus planes empresariales. Las centrales hidroeléctricas deben programar su producción mensual de energía, es por ello, que la disponibilidad de las máquinas es de suma importancia, este tipo de energía no se puede almacenar, todo lo que se produce se debe transmitir. Según los estados financieros del 2019, una planta hidroeléctrica en Colombia, con capacidad para 240 MW (megawatt) puede tener utilidades promedio de 337 millones de pesos diarios, por lo que cada disparo de alguna de las máquinas generadoras crea un impacto significativo en las finanzas de la organización. A pesar de que Las Hidroeléctricas cuenta con un grupo de planificación de mantenimientos preventivos (Mecánicos, Eléctricos y de Automatización), por el tipo de proceso de generación (denominadas a filo de agua), es impredecible saber cuándo una máquina puede presentar fallos más aun, cuando estos fallos pueden ser por diversos motivos y externos, como la calidad del agua que es un factor natural.

Las técnicas predictivas son de vital importancia, en especial cuando sirven como una herramienta de prevención, siendo posible a través del análisis de algunos comportamientos de las variables operativas de la planta (variables independientes) se puede predecir un probable fallo de una máquina (variables dependientes). Esto, permite tomar de forma rápida y eficiente las correspondientes acciones correctivas. Hoy en día, las técnicas de minería de datos, constituyen una herramienta de gran utilidad, la cual permite extraer información de una base de datos con el fin de poder encontrar modelos y técnicas que permitan visualizar con claridad la información que muestre lo que sucede con una variable especifica o deseada. Es importante resaltar que un adecuado análisis basado en técnicas de minería de datos permite definir las variables relevantes, es decir, permite establecer un filtro con el fin de seleccionar los atributos más influyentes sobre el valor de la variable objetivo (variable dependiente), antes de empezar el proceso de entrenamiento. Esto último asegura la calidad de los datos y mejora el desempeño (Castillo et al., 2011).

El uso de las técnicas inteligentes, en la predicción de fallos en Centrales Eléctricas ha sido usado desde hace algún tiempo. Actualmente, existen diversos sistemas que han sido propuestos, los cuales están basados en técnicas inteligentes como redes neuronales. Normalmente, estos sistemas son integrados a sistema expertos con el objetivo de ayudar en la toma de decisiones. Las redes neuronales (Thaeer et al., 2018) han sido empleadas, para mejorar y predecir el rendimiento de algunas hidroeléctricas, mediante técnicas de análisis de datos, logrando una predicción del 96\%. En Kougiasa et al., (2019), se analizan las principales tecnologías emergentes para el manejo de algunas fallas y otros aspectos en las centrales hidroeléctricas. Por su parte en (Rahman y Mohamad-Saleh, 2018) se estudian las técnicas de inteligencia artificial bio inspiradas, para resolver los problemas de optimización en sistemas de potencia.

Otros autores como Khan et al., (2020) proponen un sistema multiagentes para mejorar los procesos de distribución y gestión de la energía, mostrando su utilidad para minimizar los costos de interrupción, maximizar la confiabilidad, y optimizar los horarios operativos. En Teran et al., (2016), se realiza un análisis, mediante técnicas matemáticas, para predecir fallas en una central hidroeléctrica colombiana. Por su parte en Nikolić et al., (2012) realizan un modelo matemático para la optimización de diagnósticos en centrales hidroeléctricas. Este modelo, matemático selecciona los parámetros de diagnóstico, en un nivel de confiabilidad permisible. En este mismo sentido en Abdul, (2019), se utiliza la simulación Montecarlo desde el software RiskAmp de Excel, con el fin de definir la estabilidad de Taludes en una hidroeléctrica. Por su parte en Simani et al., (2015) se propone un controlador difuso, para regular la velocidad de una turbina, mediante un sistema basado en fallas para una central hidroeléctrica. En Zhu et al., (2014) se diseña un modelo para la detección de fallas en línea, por medio de componentes principales y independientes.

Hoy en día, las técnicas predictivas han sido usadas en una gran variedad de aplicaciones. En (Liu et al., 2018) se emplean técnicas micro sísmicas para prevenir el estallido de rocas en las Centrales hidroeléctricas. Así mismo, autores como Madaeni et al., (2020), muestran como estas técnicas pueden ser empleadas en diversos procesos industriales. Otros autores como Xue et al., (2020), emplean las técnicas inteligentes en la predicción del riesgo de estallido de rocas subterráneas. Autores como Hannan et al., (2020), analizan estas técnicas con el fin de Integrar diversas fuentes de energía renovable. Borunda et al., (2020), soluciona los problemas de las energías renovables (predicción, diagnóstico de fallas, mantenimiento, operación, planificación, dimensionamiento y gestión de riesgos) por medio de sistemas basados en redes neuronales, lógica difusa y redes bayesianas. Simani et al., (2016), emplea el programa Simulink de Matlab, técnicas de lógica difusa y simulación Montecarlo, para simular el control de fallas en una hidroeléctrica, por medio de la cual definen las mejores estrategias para este fin. 
Otros estudios relacionados pueden ser encontrados en: Kang et al., (2019), Zhao y Feng, (2018), Halilovič et al., (2019), Rodas y Castrillón, (2019) y Bedoya y López-Lezama, (2015), Murphy et al., (2019), Blancke et al., (2019), Contreras y Edwin, (2019) entre otros. Las diferentes revisiones bibliográficas ilustradas en esta sección muestran que si bien, se han empleado diversas técnicas en la predicción de fallos en una Central Hidroeléctrica, en estas revisiones no se encuentra un sistema de predicción de fallos basado en técnicas de Minería de datos, específicamente bajo la plataforma de aprendizaje automático y minería de datos denominado Weka (https://www.cs.waikato.ac.nz/ml/weka/). Herramienta ampliamente usada en la literatura, desarrollada por la Universidad de Waikato New Zeland (https://www.cs.waikato.ac.nz/ml/weka/index.html). En consideración a lo anteriormente planteado, surge así la siguiente pregunta de investigación: ¿Cómo diseñar una metodología para predecir la parada de máquinas en una central hidroeléctrica por medio de técnicas de minería de datos, con el fin facilitar la producción mensual y evitar pérdidas?

Finalmente, para su presentación este artículo ha sido estructurado de la siguiente manera: inicialmente, se expone la metodología empleada para abordar el problema objeto de estudio. Seguidamente, en la sección de resultados se establecen las principales causas que explican los fallos en una Central Hidroeléctrica. En la discusión de resultados, se realiza un análisis de resultados y se compara con otros trabajos similares. Finalmente, se presenta un cuerpo de conclusiones derivados de los resultados y se presenta las referencias utilizadas.

\section{MATERIALES Y METODOS}

Para la estructuración y desarrollo de la metodología, se empleó el algoritmo de clasificación bayesiana J48, ejecutado mediante la plataforma de aprendizaje automático y minería de datos denominada Weka (https://www.cs. waikato.ac.nz/ml/weka/). Este algoritmo permite predecir de forma anticipada (apriori) el fallo de una máquina (disparo), con base en las siguientes variables independientes: temperatura cojinete guía superior $\left({ }^{\circ} \mathrm{C}\right)$, flujo de agua intercambiadores de calor $\left(\mathrm{M}^{\wedge} 3 / \mathrm{Seg}\right)$, presión de regulación de velocidad (BAR), presión tubería de carga (BAR), caudal tubería de carga $\left(\mathrm{M}^{\wedge} 3 / \mathrm{Seg}\right)$, nivel de embalse $(\mathrm{MSNM})$, carga generada $(\mathrm{MW})$, frecuencia $(\mathrm{Hz})$, temperatura cojinete guía turbina $\left({ }^{\circ} \mathrm{C}\right)$, clima. Los datos fueron tomados de tres unidades generadoras cuya configuración técnica es similar y se muestra en la Tabla 1. Cada máquina de la central posee un total de 4 cojinetes los cuales son: CGS Cojinete Guía Superior), CGE (Cojinete Guía Empuje), CGI (Cojinete Guía Inferior), CGT (Cojinete Guía Turbina). La predicción de fallos, permite diseñar planes de prevención tendientes a minimizar este problema.

Para la estructuración de este documento, se propone una metodología basa en Castrillón et al., (2020). Esta metodología es organizada en cinco pasos: (1) Construcción de la base de datos; (2) análisis de correlación; (3) Diseño del archivo arff; (4) Construcción del árbol de clasificación; (5) Identificación de las principales causas de fallo.

Tabla1: Características de la maquina generadora.

\begin{tabular}{|c|c|c|c|}
\hline \multicolumn{4}{|c|}{ Especificaciones Técnicas de las Unidades Generadoras Estudiadas } \\
\hline No. De unidades & \multicolumn{3}{|l|}{3} \\
\hline Capacidad Nominal (MW) & \multicolumn{3}{|l|}{30} \\
\hline Capacidad efectiva neta (MW) & \multicolumn{3}{|l|}{19} \\
\hline Conexión al sistema & \multicolumn{3}{|c|}{ Transformadores $33 / 4.16 \mathrm{Kv}$, dos de 12,5 y $14,1 \mathrm{MVA}$} \\
\hline Características de la turbina: & Unidad No. 1 & Unidad No. 2 & Unidad No. 3 \\
\hline Tipo & hidráulico & hidráulico & hidráulico \\
\hline Fabricante & Baldwin & Baldwin & Neyrpic \\
\hline Altura de diseño (placa) (m) & 116,4 & 116,4 & 116 \\
\hline \multicolumn{4}{|l|}{ Características del generador } \\
\hline Fabricante & G. Electric & G. Electric. & Mitsubishi \\
\hline Velocidad (rpm) & 514 & 514 & 600 \\
\hline Capacidad (kva) & 11.800 & 11.800 & 14.100 \\
\hline Factor de potencia & 0.85 & 0.85 & 0.85 \\
\hline Voltaje (V) & $4.160 \mathrm{v}$. & $4.160 \mathrm{v}$. & $4.160 \mathrm{v}$. \\
\hline
\end{tabular}

Paso 1. Construcción de la base de datos: Para la construcción de la base de datos, se tomaron 300 registros directamente de los datos registrados en los últimos seis meses por los operadores de planta de la Empresa. Estos registros se captaron para cada una de las 11 variables seleccionadas. 10 variables independientes (temperatura cojinete guía superior $\left({ }^{\circ} \mathrm{C}\right)$, flujo de agua intercambiadores de calor $\left(\mathrm{M}^{\wedge} 3 / \mathrm{Seg}\right)$, presión de regulación de velocidad (BAR), presión tubería de carga (BAR), caudal tubería de carga $\left(\mathrm{M}^{\wedge} 3 / \mathrm{Seg}\right)$, nivel de embalse (MSNM), carga generada $(\mathrm{MW})$, frecuencia $(\mathrm{Hz})$, temperatura cojinete guía turbina $\left({ }^{\circ} \mathrm{C}\right)$, clima) y una variable dependiente (disparo máquina). Las tendencias estadísticas de estas variables (Mínimo, máximo, promedio y desviación estándar) también son ilustradas como resultado de este paso. 
Paso 2. Análisis de correlación: Tomando como referencia la base de datos construida en el Paso 1, se analiza la correlación (Pearson) existente entre cada una de las variables independiente respecto a la variable dependiente, con el fin de verificar que la correlación entre estas variables no sea insignificante o demasiado alta. Las variables que no cumplan con esta premisa son descartadas.

Paso 3. Diseño del archivo arff: Tomando como referencia la base de datos construida en el Paso 1 , y las variables seleccionadas en el paso 2, se construye el respectivo archivo de texto plano (encabezado y detalle), según el formato de la plataforma weka, para ser interpretado por medio del clasificador bayesiano J48, desde la plataforma de aprendizaje automático y minería de datos denominada Weka (www.cs. waikato.ac.nz/ml/weka/). En forma similar al Paso uno de la metodología, las tendencias estadísticas de estas variables (Mínimo, máximo, promedio y desviación estándar) son ilustradas en este paso.

Paso 4. Construcción del árbol de clasificación: El archivo elaborado en el paso 3, se interpreta por medio de la plataforma Weka, empleando el algoritmo de clasificación bayesiano J48, mediante la técnica de validación cruzada. Esta interpretación permite construir el respectivo árbol de decisión, sobre el cual es posible analizar las principales causas de los disparos de las máquinas. Se resalta que los algoritmos de clasificación bayesiana permiten obtener muy buenos resultados con pocos datos (Valencia et al., 2015). Como resultado de este paso, también es generada, la matriz de confusión, la precisión detallada por cada clase, donde se ilustran los resultados positivos verdaderos, falsos positivos, la medida- $F$ y los valores ROC. Valores determinantes en la precisión de los resultados. Adicionalmente, en este paso se comparan los valores obtenidos con otros algoritmos similares, con el fin de establecer el porcentaje de efectividad del mismo.

Paso 5. Identificación de las principales causas de fallo: Con base en el árbol de clasificación obtenido en el paso anterior mediante el algoritmo bayesiano $\mathrm{J} 48$, se determinan las principales causas, representadas en las variables independientes (nodos del árbol) que inciden en el disparo de las máquinas o variable dependiente (hojas del árbol).

\section{RESULTADOS}

Como consecuencia del desarrollo de cada uno de los pasos descritos en la metodología anterior, se obtuvieron los siguientes resultados:

Paso 1. Construcción de la base de datos: Para el desarrollo de esta base de datos se tomaron datos de forma aleatoria de los últimos 6 meses del 2019 sobre las variables objeto de análisis: temperatura cojinete guía superior $\left({ }^{\circ} \mathrm{C}\right.$ - TempCGS), flujo de agua intercambiadores de calor $\left(\mathrm{M}^{\wedge} 3 / \mathrm{Seg}-\right.$ FlujolC), presión de regulación de velocidad (BAR - PresionRV), presión tubería de carga (BAR - PresionTC), caudal tubería de carga $\left(\mathrm{M}^{\wedge} 3 / \mathrm{Seg}\right.$-CaudalTC), nivel de embalse (MSNM-NivelEmb), carga generada (MW - Cargagen), frecuencia (Hz-FrecHZ), temperatura cojinete guía turbina $\left({ }^{\circ} \mathrm{C}-\mathrm{TempCGT}\right)$, condiciones climáticas y disparo máquina (Dispa). De cada una de las variables se tomaron 300 registros aleatorios. Con base en estos datos se construyó la Tabla 2. Igualmente, en este punto se adiciona la Tabla 3, con el fin de mostrar las tendencias estadísticas de cada una de las variables analizadas, en la tabla 2.

Tabla 2: Selección de datos de variables seleccionadas. Por razones de espacio no se muestran todos los datos

\begin{tabular}{|l|l|l|l|l|l|l|l|l|l|l|}
\hline $\begin{array}{l}\text { Temp } \\
\text { CGS }\end{array}$ & $\begin{array}{l}\text { Flujo } \\
\text { IC }\end{array}$ & $\begin{array}{l}\text { Pres } \\
\text { RV }\end{array}$ & $\begin{array}{l}\text { Pres } \\
\text { TC }\end{array}$ & $\begin{array}{l}\text { Caud } \\
\text { TC }\end{array}$ & $\begin{array}{l}\text { Nivel } \\
\text { Emb }\end{array}$ & $\begin{array}{l}\text { Carga } \\
\text { Gen }\end{array}$ & $\begin{array}{l}\text { Frec } \\
\text { HZ }\end{array}$ & $\begin{array}{l}\text { Temp } \\
\text { CGT }\end{array}$ & $\begin{array}{l}\text { Condiciones } \\
\text { Climáticas }\end{array}$ & Dispa \\
\hline 50 & 115 & 30 & 11 & 6 & 1323.28 & 7 & 60 & 47 & cálido & NO \\
\hline 52 & 112 & 29 & 11 & 7 & 1323.34 & 6 & 60 & 45 & nublado & NO \\
\hline$\ldots$ & $\ldots$ & $\ldots$ & $\ldots$ & $\ldots$ & $\ldots$ & $\ldots$ & $\ldots$ & $\ldots$ & $\ldots$ & $\ldots$ \\
\hline 62 & 105 & 26 & 11 & 6 & 1323.04 & 5 & 60 & 43 & Iluvia fuerte & SI \\
\hline
\end{tabular}

Tabla 3: Valores estadísticos de las variables Ilustradas en la Tabla 2.

\begin{tabular}{|l|l|l|l|l|}
\hline Variable & Mínimo & Máximo & Promedio & Desviación \\
\hline Temp CGS & 48 & 75 & 59.63 & 7.955 \\
\hline Flujo IC & 72 & 120 & 100.05 & 13.639 \\
\hline PresionRV & 25 & 31 & 27.717 & 2.078 \\
\hline PresionTC & 10 & 11 & 10.95 & 0.218 \\
\hline CaudalTC & 5 & 8 & 6.317 & 1.206 \\
\hline Nivel Emb & 1323 & 1323.97 & 1323.497 & 0.275 \\
\hline CargaGen & 5 & 7 & 5.8 & 0.814 \\
\hline FrecHz & 60 & 61 & 60.117 & 0.322 \\
\hline Temp CGT & 40 & 55 & 45.783 & 3.348 \\
\hline Condiciones Climáticas & $\mathrm{Cálido}=110$ & Nublado $=60$ & Lluvia fuerte $=55$ & Lluvia moderada $=75$ \\
\hline Disparo & $\mathrm{Si}=155$ & No $=145$ & & \\
\hline
\end{tabular}


Paso 2. Análisis de Correlación: Por medio del programa Excel, se estableció la correlación (Pearson) entre cada una de las variables independientes respecto a la variable dependiente como se ilustra en la Tabla 4. Como resultado de este análisis, las variables que presentan una correlación baja o muy alta (variables indicadas con un * en la Tabla 3.) son suprimidas, seleccionándose solo aquellas variables que presentan una correlación moderada, respecto a la variable dependiente.

Tabla 4: Correlaciones variables independientes vs variable dependiente

\begin{tabular}{|l|c|}
\hline Variable Independiente & $\begin{array}{l}\text { Correlación Variable } \\
\text { Dependiente }\end{array}$ \\
\hline Temperatura (Cojinete guía superior) $^{*}$ & 0.16 \\
\hline Flujo de agua $^{*}$ & 0.13 \\
\hline Presión Regulador de velocidad & 0.24 \\
\hline Presión tubería de carga & 0.22 \\
\hline Caudal Tubería de carga & 0.71 \\
\hline Nivel de embalse* & 0.02 \\
\hline Carga generada & 0.73 \\
\hline Frecuencia $^{*}$ & 0.35 \\
\hline Temperatura (Cojinete guía turbina) $^{*}$ & 0.07 \\
\hline Clima $^{*}$ & 0.14 \\
\hline
\end{tabular}

Paso 3. Diseño del archivo arff: Con el fin de analizar los datos desde la plataforma de aprendizaje automático y minería de datos denominada Weka (https://www.cs.waikato.ac.nz/ml/weka/), se toman las variables seleccionadas en el paso anterior y se estructura el respectivo archivo arff el cual podrá ser interpretado por esta plataforma. La estructura de este archivo, se muestra en dos tablas denominadas encabezado y datos, como se lustra a continuación en las Tablas 5 y 6 . Las tendencias de cada una de las variables independientes analizadas en la Tabla 6, fueron ilustradas en la Tabla 3.

Tabla 5: Encabezado del archivo Arff

\begin{tabular}{|l|l|}
\hline Nombre campo & Tipo de dato \\
\hline @ATTRIBUTE PresionRV & Integer \\
\hline @ATTRIBUTE PresionTC & Integer \\
\hline @ATTRIBUTE CaudalTC & Integer \\
\hline @ATTRIBUTE CargaGen & Integer \\
\hline @ATTRIBUTE FrecHz & Integer \\
\hline @ATTRIBUTE Disparo & $\{$ "SI","NO"\} \\
\hline
\end{tabular}

Tabla 6: Datos del archivo Arff. Por razones de espacio no se muestran todos los datos

\begin{tabular}{|l|l|l|l|l|l|}
\hline PresionRV & PresionTC & CaudalTC & CargaGen & FrecHZ & Disparo \\
\hline 30 & 11 & 6 & 7 & 60 & NO \\
\hline 27 & 11 & 8 & 7 & 60 & NO \\
\hline 29 & 11 & 7 & 6 & 60 & NO \\
\hline$\ldots$ & $\ldots$ & $\ldots$ & $\ldots$ & $\ldots$ & $\ldots$ \\
\hline 29 & 11 & 6 & 5 & 60 & SI \\
\hline 31 & 10 & 5 & 5 & 60 & SI \\
\hline
\end{tabular}

Paso 4. Construcción del árbol de clasificación: Con base en el archivo Arff generado en el paso anterior, se construyó el respectivo árbol de clasificación para ser interpretado por medio del algoritmo Bayesiano J48. Este árbol es interpretado en la Figura 1.

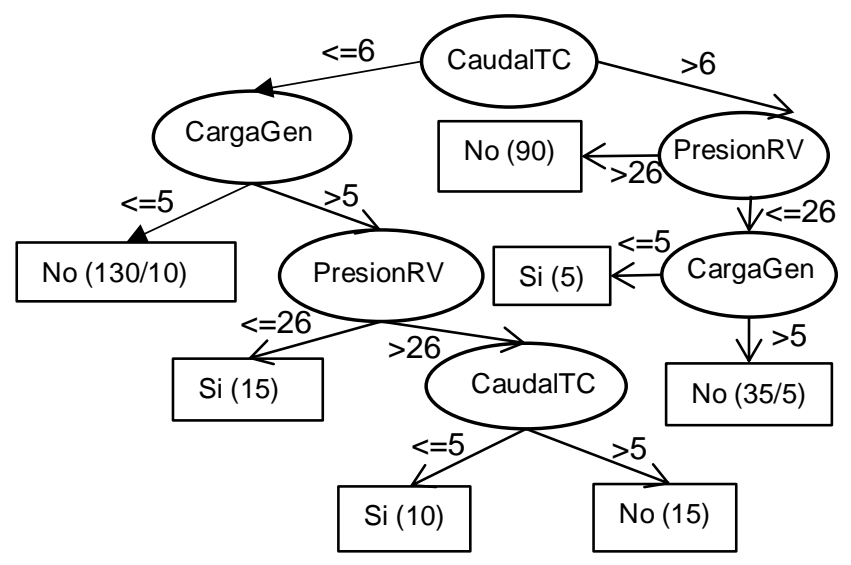

Fig. 1: Árbol de clasificación 
La clasificación lograda por este algoritmo, permite un nivel de aciertos del 94\%, mediante un proceso de validación cruzada. Las tablas 7 al 9 muestran los resultados. La Tabla 7 presenta los resultados generados por el WEKA, la Tabla 8 muestra la respectiva matriz de confusión y la Tabla 9 entrega la precisión detallada por clase.

Tabla 7: Resultados generados por el WEKA

\begin{tabular}{|l|l|l|}
\hline Variable & Valor & $\%$ \\
\hline Correctly Classified Instances & 282 & $94 \%$ \\
\hline Incorrectly Classified Instances & 18 & $6 \%$ \\
\hline Kappa statistic & 0.88 & \\
\hline Mean absolute error & 0.09 & \\
\hline Root mean squared error & 0.23 & \\
\hline Relative absolute error & $18.38 \%$ & \\
\hline Root relative squared error & $45.80 \%$ & \\
\hline Total Number of Instances & 300 & \\
\hline
\end{tabular}

Tabla 8: Matriz de Confusión

\begin{tabular}{|l|l|l|}
\hline$a$ & $b$ & classified as \\
\hline 147 & 8 & $\mathrm{a}=\mathrm{SI}$ \\
\hline 10 & 135 & $\mathrm{~b}=\mathrm{NO}$ \\
\hline
\end{tabular}

Tabla 9: Precisión detallada por clase

\begin{tabular}{|l|l|l|l|l|l|l|}
\hline Positivos Verdaderos & Falsos Positivos & Precision & Repetición & Medida $-F$ & ROC Area & Clase \\
\hline 0.948 & 0.069 & 0.936 & 0.948 & 0.942 & 0.965 & SI \\
\hline 0.931 & 0.052 & 0.944 & 0.931 & 0.938 & 0.965 & NO \\
\hline Weighted Avg. & 0.940 & 0.061 & 0.94 & 0.94 & 0.94 & 0.965 \\
\hline
\end{tabular}

Finalmente, en este punto se muestra como, empleando otras técnicas de clasificación, se pueden obtener resultados similares, sin embargo, no todas estas técnicas ilustradas en la Tabla 10, permiten obtener un árbol de clasificación, claro como el mostrado en la Figura 1. Siendo este un aspecto de vital importancia cuando se desean diseñar políticas tendientes a reducir este problema en una Central Hidroeléctrica.

Tabla 10: Comparación con otras técnicas similares

\begin{tabular}{|l|l|l|}
\hline Técnica & Acierto & Referencia \\
\hline ADtree & $93.33 \%$ & https://weka.sourceforge.io/doc.stable/weka/classifiers/trees/ADTree.html \\
\hline DecisionStump & $88.33 \%$ & https://weka.sourceforge.io/doc.stable-3-8/weka/classifiers/trees/DecisionStump.html \\
\hline Ft & $92.66 \%$ & https://weka.sourceforge.io/doc.packages/functionalTrees/weka/classifiers/trees/FT.html \\
\hline J48Graft & $94 \%$ & https://weka.sourceforge.io/doc.packages/J48graft/weka/classifiers/trees/J48graft.html \\
\hline LADTree & $94 \%$ & https://weka.sourceforge.io/doc.stable/weka/classifiers/trees/LADTree.html \\
\hline RandomTree & $95 \%$ & https://weka.sourceforge.io/doc.dev/weka/classifiers/trees/RandomTree.html \\
\hline Reptree & $94 \%$ & https://weka.sourceforge.io/doc.dev/weka/classifiers/trees/REPTree.html \\
\hline
\end{tabular}

Paso 5. Identificación de las principales causas de fallo: Se identifican tres situaciones en las cuales se puede producir un disparo de las máquinas, cada una de estas situaciones está marcada por las diversas relaciones de causas, que son ilustradas en la Tabla 11, donde se identifican tres principales causas de disparo.

Tabla 11: Diferentes relaciones de causa

\begin{tabular}{|l|l|l|l|l|}
\hline Var Independiente 1 & Var Independiente 2 & Var Independiente 1 & Var Independiente 1 & Var dependiente \\
\hline CaudalTC $<=6 \rightarrow$ & Cargagen $>5 \rightarrow$ & PresionRv $<=26 \rightarrow$ & & Disparo \\
\hline CaudalTC $<=6 \rightarrow$ & Cargagen $>5 \rightarrow$ & PresionRv $>=26 \rightarrow$ & CaudalTC $<=5 \rightarrow$ & Disparo \\
\hline CaudalTC $>6 \rightarrow$ & PresionRV $<=26 \rightarrow$ & Cargagen $<=5 \rightarrow$ & & Disparo \\
\hline
\end{tabular}

\section{DISCUSION}

En el árbol de decisión, ilustrado en la Figura 1, se puede evidenciar un ordenamiento ascendente de la importancia de las variables en el disparo de la máquina. En el primer nivel se identifica la variable Caudal tubería carga (CaudalTC), cuando este caudal es menor o igual a $6 \mathrm{~m} 3 / \mathrm{min}$, la máquina tiene el caudal justo para operar ya que por el diseño de la máquina se requiere de una fuerza mínima necesaria para hacer girar el rodete de la turbina, si esta fuerza es inferior, la máquina no podrá operar. Las otras variables influyentes en este proceso son la presión del regulador de velocidad y Carga generada. La primera de estas variables (Presión de regulador de velocidad) muestra que cuando la presión es menor a 26 bares, la máquina se dispara. La presión que se mide en esta parte corresponde al sistema hidráulico del regulador de velocidad, 
este sistema afecta de forma directa algunas variables del grupo del generador como son la Potencia Activa, Potencia Reactiva, Frecuencia, Tensión Terminal y Posición del Distribuidor, por ende, la importancia de que este opere de manera estable.

Sin la presurización del sistema hidráulico, que en este caso es de aceite con un acumulador de gas (nitrógeno) tipo vejiga marca REIVAX, el sistema no sería capaz de mover los brazos axiales que se encargan de abrir los distribuidores de regulación de la velocidad, lo cual hace que la frecuencia nominal de la máquina no sea constante, impidiéndole a la máquina conectarse a la red (Sincronización). Es importante resaltar que para que la máquina pueda liberar energía a la red debe estar sincronizada, es decir estar en la misma frecuencia, nivel de tensión y ángulo de fase de la red, de lo contrario la máquina se desconecta de la red parándose. La otra variable Carga generada (Cargagen) constituye un excelente indicador de estabilidad en la operación.

Un análisis de literatura descrita en este trabajo (Thaeer et al., 2018; Kougiasa et al., 2019; Teran et al., 2016, entre otros) muestra que no existe un análisis de fallos de máquinas, estructurado con esta técnica de minería de datos, bajo un nivel de precisión igual o superior al 94\%, como se ilustra en este documento. Finalmente, es importante resaltar que los resultados son válidos en el caso de estudio analizado, por lo cual es necesario extender la aplicación de la metodología a otras empresas generadoras de electricidad. Igualmente, como futuras líneas de investigación se propone incluir algunos otros componentes como: regulador de velocidad, regulador de voltaje, bombas, caballetes, polos, corrientes de cortocircuito, transformador, niveles de aceite de cojinetes y transformador, zapatas, caracol, esférica, entre otros.

\section{CONCLUSIONES}

Por medio del árbol de decisión se puede observar de forma clara y concisa las variables más importantes que pueden causar un paro de una máquina, estas variables son: Caudal de tubería de carga, presión de regulación de velocidad y carga generada. En los procesos de generación energética lo ideal es tener funcionando las máquinas $24 \mathrm{~h}$ al día 7 días a la semana todos los meses del año. Lo anterior permitirá cumplir los pronósticos de oferta. Este cumplimiento de pronósticos, protege a la empresa de incurrir en infracciones, las cuales son penalizadas con altas sumas de dinero. Facilitar el cumplimiento de estos pronósticos de ofertas y minimizar el costo de disparo de una máquina, al reducir sus paradas, son dos aspectos de vital importancia logrados en esta investigación.

El árbol de clasificación obtenido, en esta investigación, permite obtener una mirada mucho más clara de la información objeto de análisis. Este árbol, permite predecir fácilmente, futuros paros de máquinas con un acierto superior al 90\% (exactamente 94\%). La correcta predicción del disparo de una máquina, dependerá de los eventos que se presenten, y de su posible corrección de forma oportuna, evitando pérdidas de generación y posibles detrimentos para la empresa. Trabajos similares a este, podrían ser aplicados en la predicción de diferentes tipos de fallos, en diversas empresas y máquinas. Finalmente, se resalta que replicar este trabajo es fácil, para ello solo se debe seguir cada uno de los pasos estructurados en la metodología definida en este trabajo investigativo, algo más factible de lograr si se tiene presente que esta investigación está desarrollada bajo la plataforma aprendizaje automático y minería de datos denominada Weka.

\section{AGRADECIMIENTOS}

Se agradece la colaboración a la Universidad Nacional de Colombia Sede Manizales y en especial al Departamento de Ingeniería Industrial de esta Sede.

\section{REFERENCIAS}

Abdul, N., Investigation on Slope Stability using Monte Carlo Simulation: A Case Study of Ulu Jelai Hydroelectric Project Slope Parameters \& Design Concept, Materials Today: Proceedings, ISSN: 2214-7853, 19, 1216-1224 (2019)

Bedoya, V., y López-Lezama, J., Modelo para el Control de Inundaciones durante el Fenómeno De "La Niña" Utilizando un Embalse Hidroeléctrico, doi: 10.4067/S0718-07642015000200011, Información Tecnológica, 26(2), 89-100 (2015)

Blancke, O., Tahan, A., y otros 4 autores más, A Holistic Multi-Failure Mode Prognosis Approach for Complex Equipment, https://doi.org/10.1016/j.ress.2018.07.006, Reliability Engineering and System Safety, 180, 136-151 (2018)

Borunda, M., Jaramillo, O.A., y Otros dos autores más, Bayesian Networks in Renewable Energy Systems: $A$ Bibliographical Survey, http://dx.doi.org/10.1016/j.rser.2016.04.030, Renewable and Sustainable Energy Reviews, 62, 32-45 (2016)

Castillo, C., Mendoza, M., y Poblete, B., Information Credibility on Twitter, https://doi.org/10.1145/1963405.1963500, WWW '11 Proceedings of the 20th International Conference on World Wide Web, 675-684 (2011) 
Castrillón, O., Sarache, W., y Ruiz, S., Predicción del Rendimiento Académico por medio de Técnicas de Inteligencia Artificial, http://dx.doi.org/10.4067/S0718-50062020000100093, Formación Universitaria, 13(1), 93-102 (2020)

Contreras, L-F., y Edwin, T.B., Slope Reliability and Back Analysis of Failure with Geotechnical Parameters Estimated Using Bayesian Inference, https://doi.org/10.1016/j.jrmge.2018.11.008, Journal of Rock Mechanics and Geotechnical Engineering, 11, 628e643 (2019)

Halilovič, M., Urevc, J., y Koc P., Prediction of Recirculation Flow Rate for Icing Prevention in Water Intake Supply Systems of Nuclear Power Plants, https://doi.org/10.1016/j.coldregions.2019.02.013, Cold Regions Science and Technology, 161, 63-70 (2019)

Hannan, M.A., Tan, S., y Otros tres autores más, Optimized Controller for Renewable Energy Sources Integration into Microgrid: Functions, Constraints and Suggestions, https://doi.org/10.1016/j.jclepro.2020.120419, Journal of Cleaner Production, 256, 120419 (2020)

Kang, F., Li, J., y Dai. J., Prediction of Long-Term Temperature Effect in Structural Health Monitoring of Concrete Dams Using Support Vector Machines with Jaya Optimizer and Salp Swarm Algorithms, https://doi.org/10.1016/j.advengsoft.2019.03.003, Advances in Engineering Software, 131, 60-76 (2019)

Khan, M., Wang, J., y Otros 4 autores más, Optimal Energy Management and Control Aspects of Distributed Microgrid Using Multi-Agent Systems, https://doi.org/10.1016/j.scs.2018.11.009, Sustainable Cities and Society, 44, 855-870 (2019)

Kougiasa, I., Aggidisb, G., y otros 10 autores, Analysis of Emerging Technologies in the Hydropower Sector, https://doi.org/10.1016/j.rser.2019.109257, Renewable and Sustainable Energy Reviews, 113, 109257 (2019)

Liu, F., Ma., T., y otros dos autores, Prediction of Rockburst in Tunnels at the Jinping II Hydropower Station Using Microseismic Monitoring Technique, https://doi.org/10.1016/j.tust.2018.08.010, Tunnelling and Underground Space Technology, 81, 480-493 (2018)

Madaeni, F., Lhissou, R., y tres autores más, Ice Jam Formation, Breakup and Prediction Methods Based on Hydroclimatic Data Using Artificial Intelligence: A Review, https://doi.org/10.1016/j.coldregions.2020.103032, Cold Regions Science and Technology, 174, 103032 (2020)

Murphy, S., Sowell, F., y Apt, J., A Time-Dependent Model of Generator Failures and Recoveries Captures Correlated Events and Quantifies Temperature Dependence, https://doi.org/10.1016/j.apenergy.2019.113513, Applied Energy, 253, 113513 (2019)

Nikolić, D., Nikolić, R.R., y Cinco autores más, Optimization of Technical Diagnostics Procedures for Hydroelectric Power Plants, doi: 10.1016/j.proeng.2012.07.102, Procedia Engineering, 40, 322 - 327 (2012)

Rahman, I., y Mohamad-Saleh, J., Hybrid Bio-Inspired Computational Intelligence Techniques for Solving Power System Optimization Problems: A Comprehensive Survey, https://doi.org/10.1016/j.asoc.2018.04.051, Applied Soft Computing, 69, 72-130 (2018)

Rodas, L., y Castrillón, O., Predicción de Fallos Mecánicos en Equipos de Envoltura, http://dx.doi.org/10.4067/S071807642019000600111, Información Tecnológica, 30(6), 111-122 (2019)

Simani, S., Alvisi, S., y Venturini, M., Fault Tolerant Control of a Simulated Hydroelectric System, http://dx.doi.org/10.1016/j.conengprac.2016.03.010, Control Engineering Practice, 51, 13-25 (2016)

Simani, S., Alvisi, S., y Venturini, M., Data-Driven Design of a Fault Tolerant Fuzzy Controller for a Simulated Hydroelectric System, IFAC-PapersOnLine, ISSN: 2405-8963, 48-21 y 1090-1095 (2015)

Teran, L.A., Roa, C.V., y seis autores más, Failure Analysis of a Run-of-the-river Hydroelectric Power Plant, http://dx.doi.org/10.1016/j.engfailanal.2016.05.035, Engineering Failure Analysis, 68, 87-100 (2016)

Thaeer, A., Bin Sulaiman, M., y Abdalla, A., Prediction of Small Hydropower Plant Power Production in Himreen Lake Dam (HLD) Using Artificial Neural Network, https://doi.org/10.1016/j.aej.2016.12.011, Alexandria Engineering Journal, 57(1), 211-221 (2018)

Valencia, M., Correa, J., y Díaz, F., Métodos Estadísticos Clásicos y Bayesianos para el Pronóstico de Demanda. Un Análisis Comparativo, https://doi.org/10.15446/rev.fac.cienc.v4n1.49775, Revista Facultad de Ciencias Universidad Nacional de Colombia, 4(1), 52 -67 (2015)

Xue, Y., Bai, C., y Otros cinco autores más, A Two-step Comprehensive Evaluation Model for Rockburst Prediction Based on Multiple Empirical Criteria, https://doi.org/10.1016/j.enggeo.2020.105515, Engineering Geology, 268, 105515 (2020)

Zhao, J., y Feng, X., Microseismicity Monitoring and Failure Mechanism Analysis of Rock Masses with Weak Interlayer Zone in Underground Intersecting Chambers: A Case Study from the Baihetan Hydropower Station, China, https://doi.org/10.1016/j.enggeo.2018.08.006, Engineering Geology, 245, 44-60 (2018)

Zhu, W., Zhou, J., y cuatro autores más, A novel KICA-PCA Fault Detection Model for Condition Process of Hydroelectric Generating Unit, http://dx.doi.org/10.1016/j.measurement.2014.08.026, Measurement, 58, 197-206 (2014) 